\title{
An Efficient Heuristics for Minimizing Total Holding Cost with no Tardy Jobs in Job Shop Scheduling
}

\author{
G. Ramya and M. Chandrasekaran
}

\begin{abstract}
Challenging combinatorial optimization problems are encountered even in the job shop scheduling problems. This paper works reveals with minimizing the total holding cost of completed and in-process products subject to no tardy jobs with Sheep Flocks Heredity Model Algorithm (SFHM) and Shuffled Frog Leaping Algorithm (SFLA) for job shop scheduling problem (JSP). Heuristics algorithms are developed in scholastic search way in which natural big buoyancy is maintained with respect to optimum schedule. Results are produced and compared with literature results in terms of total holding cost, stipulated time and computational time. SFL algorithm performs result oriented than other Heuristics Algorithm.
\end{abstract}

Index Terms-Job Shop scheduling, shuffled frog leaping algorithm, sheep flocks heredity model algorithm and total holding cost.

\section{INTRODUCTION}

A schedule is an allocation of tasks to the time intervals on the machines. The aim is to find a schedule that minimizes the overall completion time, which is called the makespan. In the job shop scheduling problem $n$ jobs have to be processed on $\mathrm{m}$ different machines. Each job consists of a sequence of tasks that have to be processed during an uninterrupted time period of a fixed length on a given machine. Due dates are treated as deadlines and completion of job in a given stipulated time if delayed, penalties, bad impression, loss of future sales and rush shipping cost are negative images been created.

To avoid this, the job-shop scheduling problem has been extensively studied with the objective of minimizing some functions of the completion times of jobs. Several techniques have been proposed and different heuristics have been designed and developed for solving the minimum makespan problem, the minimum total tardiness problem and so on. SFHM algorithm was used for minimizing mean tardiness and mean flow time multi objective criteria [1]. An effective SFLA was used for minimizing maximum completion time (i.e., makespan) [2]. In this work SFLA and SFHM algorithm are used for solving the scheduling problem to meet due dates in a simple job shop. It is developed to approximately minimize the total holding cost which corresponds to the sum of product inventory cost and in-process inventory cost. Several benchmark problems are solved by the proposed

Manuscript received August 2, 2012; revised October 8, 2012.

F. G. Ramya is with the Sathyabama University, Chennai, Tamil Nadu, CO 600119 India (e-mail: ramyacadcam@gmail.com).

S. M. Chandrasekaran is with Vels University, Chennai, Tamil Nadu, CO 600117 India (e-mail: ch_sekaran@yahoo.com). algorithms and the results are compared with literature results.

\section{JOB SHOP SCHEDULING FOR MinimizING TOTAL HOLDING COST SUBJECT TO NO TARDY JOBS [3]}

\section{A. Problem Description}

A set of I jobs has to be processed on $\mathrm{K}$ machines. Let $O^{l}{ }_{i}$ $\left(l=1,2, \ldots, L_{i}\right)$ denote the $l^{\text {th }}$ operation of job $J_{i}$, where $L_{i}$ corresponds to the number of operations for job $J_{i}$. The processing time $p_{i}^{l}$ of operation $O^{l}{ }_{i}$ is pre-specified. The due date $\mathrm{d}_{\mathrm{i}}$ of job $J_{i}$ is pre-specified by the associated customer. The shop incurs the holding cost for in-process time once a job begins processing, and if a job is completed earlier than its due date, then the shop holds the job and incurs the holding cost for earliness. Assume $w^{l-1}{ }_{\mathrm{i}}<w_{i}^{l}$, where $w_{i}^{l}(l=1$, $\left.2, \ldots, L_{i-1}\right)$ denotes the holding cost per unit time for in-process product in idle time from end of operation $O^{l}{ }_{i}$ to start of operation $O^{l+1}{ }_{i}$, and $w^{L i}{ }_{i}$ denotes the holding cost per unit time for completed product from end of operation $O^{L i}{ }_{i}$ to due date $d_{i}$. Let $C^{m}{ }_{i}$ (decision variable) denote the completion time of operation $O^{m}{ }_{i}$ and $E_{k}$ the set of operations to be performed on machine $k$, then the problem is as follows: Eq. (2) and (3) are the conjunctive and disjunctive constraints, respectively. Eq. (4) is the due-date constraint, and Eq. (5).

$$
1 \quad L_{i}-1
$$

Minimize $f=\Sigma\left\{\sum w_{i}^{l}\left(C^{l+1}{ }_{i}-p^{l+1}{ }_{i}-C_{i}^{l}\right)+w^{L i}{ }_{i}\left(d_{i}-C^{L i}{ }_{i}\right)\right\}$ $i=1 l=1$

Subject to

$$
\begin{gathered}
C_{i}^{m}-C^{m-1}{ }_{i} \geq p^{m}{ }_{i}, \quad i=1, \ldots ., \mathrm{I}, m=2, \ldots, L_{i}, \\
C^{m}{ }_{i}-C^{n}{ }_{i} \geq p^{m}{ }_{i} V C^{n}{ }_{j}-C^{m}{ }_{j} \geq p_{j}^{n}, \\
d_{i}-C^{L i}{ }_{i} \geq 0, i=1 \\
C_{i}^{l}-p_{i}^{l} \geq 0, i=1
\end{gathered}
$$

\section{B. Proposed Heuristics Methodologies}

The heuristic approaches were also employed for various engineering application problems due to their robustness and convergence to global optima. Heuristic method of learning involves discovery and problem solving using reasoning and past experience. An approach without formal guarantee of performance can be considered a "heuristic". These heuristic approaches are used in practical situation when no better methods are available. The following section deals with the proposed heuristic approaches like Shuffled Frog leaping Algorithm and Sheep Flock Heredity Model Algorithm.

\section{Shuffled Frog Leaping Algorithm}

SFLA for solving the JSS problem with minimizing total holding cost and makespan criterion are proposed by 
population initialization, partitioning scheme, memetic evolution process, shuffling process, and a local search. SFLA was combination of memetic Algorithm and Particle Swarm Optimization. It has been performed from memetic evolution of a group of frogs when seeking for food. The initial population of frogs was partitioned into groups or subsets called "memeplexes" and the number of frogs in each subset was equal.

The SFLA was follows two search techniques compiling think globally and act locally searching. Each operation is decided by meeting pre-specified due dates and minimizing objective function. Based on local search to reach the makespan, the frogs in each subset improve their positions to have more foods. Each operation is decided by meeting pre-specified due dates and minimizing objective function.

\section{Sheep Flock Heredity Model Algorithm}

Normally, sheep in an each flock are living within their own flock under the control of shepherds. So, the genetic inheritance only occurs within the flock in other words, some special characteristics in one flock develop only within the flock by heredity, and the sheep with high fitness characteristics to their environment breed in the flock. In such a world, let us assume that two sheep flocks were occasionally mixed in a moment when shepherds looked [4]. Therefore, several sheep of one flock are inevitably mixed with the other flocks.

In sheep flocks heredity model algorithm special string structure, hierarchical genetic operations (crossover and mutation) are introduced. They are (1) sub-chromosome level genetic operation and (2) chromosome (global) level genetic operation. Implementation of Heuristics and finding results in terms of total holding cost and makespan for 10-machines $\mathrm{X} 10$ jobs Lawrence (LA16) Problem [5] was as follows.

\section{IMPLEMENTATION OF SFL ALGORITHM}

Initial population of sequence generated randomly by increasing order and selected sequence divided into number of memeplexes.

\section{A. Local Search Process}

The division is done with the high level frog (column sequence) arranged in first memeplex, second one arranged in second memeplex, the last frog to the last memeplex and repeated frog back to the next order memeplex. Fitness function evaluated within the limits that the memeplex are infeasible.

\section{B. Global information Exchange Process}

The best frog memeplex values were identified each subset was compared to each other to produce best sequence way of schedule. For each iteration the frogs with the best fitness and worst fitness were identified and also the frog with the makespan schedule was identified. Finally, if the convergence criteria are not satisfied the position of the worst frog for the memeplex is adjusted and new subsets of memeplex will be created for the next iteration. This procedure is repeated for desired number of iterations to reach optimal results shown in Table I.
TABLE I: ITERATION RESULTS FOR SFLA

\begin{tabular}{|c|c|c|}
\hline & \multicolumn{2}{|c|}{ Local Search } \\
\hline Iteration's & Makespan & Total holding cost (Rs) \\
\hline First & 944 & 151798.6 \\
\hline Second Iteration & 942 & 157548.6 \\
\hline Last & 940 & 150365.6 \\
\hline Global Information Exchange \\
\hline Iteration's & Makespan & Total holding cost (Rs) \\
\hline First & 944 & 151798.6 \\
\hline Second Iteration & 945 & 152473.6 \\
\hline Last & 939 & 150268.6 \\
\hline
\end{tabular}

\section{IMPLEMENTATION OF SFHM ALGORITHM}

Initial sequence generated randomly and the corresponding makespan for the sequence is given Table II and final sequence is given in Table III.

TABLE II: INITIAL SEQUENCE

\begin{tabular}{|l|l|l|l|l|l|l|l|l|l|}
\hline 7 & 8 & 6 & 9 & 4 & 10 & 5 & 1 & 3 & 2 \\
\hline 7 & 9 & 2 & 4 & 1 & 6 & 5 & 2 & 3 & 8 \\
\hline 9 & 10 & 7 & 5 & 8 & 4 & 1 & 10 & 6 & 3 \\
\hline 9 & 1 & 5 & 7 & 3 & 10 & 8 & 2 & 6 & 4 \\
\hline 2 & 3 & 7 & 9 & 8 & 10 & 1 & 5 & 4 & 6 \\
\hline 1 & 4 & 3 & 7 & 9 & 5 & 8 & 10 & 6 & 2 \\
\hline 6 & 10 & 5 & 1 & 9 & 7 & 4 & 2 & 3 & 8 \\
\hline 9 & 2 & 5 & 3 & 1 & 6 & 8 & 10 & 7 & 4 \\
\hline 3 & 6 & 8 & 4 & 2 & 9 & 10 & 7 & 5 & 1 \\
\hline 7 & 8 & 6 & 9 & 4 & 10 & 5 & 1 & 3 & 2 \\
\hline
\end{tabular}

TABLE III: FINAL SEQUENCE

\begin{tabular}{|l|l|l|l|l|l|l|l|l|l|}
\hline 7 & 8 & 3 & 4 & 9 & 6 & 5 & 2 & 10 & 1 \\
\hline 5 & 7 & 4 & 2 & 1 & 3 & 9 & 8 & 6 & 10 \\
\hline 7 & 9 & 1 & 2 & 4 & 10 & 8 & 6 & 3 & 5 \\
\hline 1 & 9 & 7 & 5 & 8 & 3 & 4 & 6 & 10 & 2 \\
\hline 8 & 4 & 3 & 6 & 1 & 2 & 9 & 7 & 10 & 5 \\
\hline 3 & 10 & 9 & 7 & 6 & 4 & 2 & 5 & 8 & 1 \\
\hline 7 & 5 & 9 & 8 & 1 & 6 & 2 & 10 & 4 & 3 \\
\hline 5 & 10 & 7 & 2 & 6 & 4 & 8 & 3 & 9 & 1 \\
\hline 4 & 6 & 1 & 3 & 7 & 5 & 10 & 9 & 8 & 2 \\
\hline 6 & 2 & 9 & 3 & 8 & 10 & 5 & 1 & 7 & 4 \\
\hline
\end{tabular}

Makespan $=945 \mathrm{sec} \quad$ THC $=$ Rs $155458($ where $\mathrm{t}=1.8)$

\section{A. Process of Sub Chromosome Level Inverse Mutation}

The Probability for this chromosome is less than process mutation probability. Each sub string 4 and 9 positions are chosen randomly to perform inverse mutation. 


\section{B. Global Level Crossover Process}

The Crossover probability this sting is less than the process crossover probability. Sequences 1 and 6 are selected for crossover. At the position of $6^{\text {th }}$ sub string is chosen as the crossover position.

\section{Global Level Inverse Mutation Process}

The Probability for this string is less than process mutation probability. The $2^{\text {nd }}$ and $6^{\text {th }}$ sub string positions are randomly selected to perform inverse mutation.

\section{RESUlTS AND DisCUSSIONS}

Results were compared with the existing Shifting Bottleneck (SB) procedure and Artificial Immune System (AIS) reported and formulated for the minimum makespan problem, the holding cost and a due date for each job was added [5]. Computation time is calculated and the best solution for each case shown in Table IV and Fig. 1.

TABLE IV: COMPARISON RESULTS OF HEURISTICS WITH AIS AND SB PROCEDURE

\begin{tabular}{|c|c|c|}
\hline Time(t) & Best Value (Rs) & CPU time in sec \\
\hline \multicolumn{3}{|c|}{ SFHM algorithm } \\
\hline 1.8 & 155458 & 14 \\
\hline 1.9 & 162765 & 11.5 \\
\hline 2.5 & 181715 & 19.6 \\
\hline 3.5 & 219945 & 11.5 \\
\hline \multicolumn{3}{|c|}{ SFL algorithm } \\
\hline 1.8 & 155459 & 14.5 \\
\hline 1.9 & 161003 & 10.9 \\
\hline 2.5 & 176458 & 17 \\
\hline 3.5 & 216400 & 11.2 \\
\hline \multicolumn{3}{|c|}{ AIS algorithm } \\
\hline 1.8 & 160112 & 25 \\
\hline 1.9 & 164354 & 18 \\
\hline 2.5 & 184679 & 29.5 \\
\hline 3.5 & 220567 & 15.7 \\
\hline \multicolumn{3}{|c|}{ SB algorithm } \\
\hline 1.8 & 160374 & 28.2 \\
\hline 1.9 & 165402 & 25.2 \\
\hline 2.5 & 186995 & 32.7 \\
\hline 3.5 & 221604 & 19.1 \\
\hline
\end{tabular}

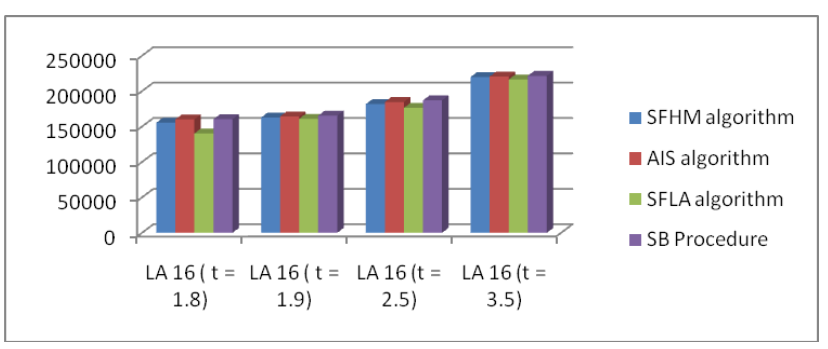

Fig. 1. Comparison values of Total Holding Cost with literature results.

\section{CONCLUSION}

This paper has proposed SFL algorithm and SFHM algorithm for the job shop scheduling to minimize total holding cost of completed and in-process products subject to no tardy jobs. The SFL algorithm is based on memetic evolution process. It solves Lawrence benchmark problems within the ready-time and due-date constraints. Computation results indicate that the proposed SFL algorithm performs well especially on the problem with tight and loose due dates. The computational time and holding cost is less when compared with AIS algorithm and literature results.

\section{REFERENCES}

[1] M. Chandrasekaran, P. Asokan, S. Kumanan, S. U. Maheshwari, "Multi objective optimization for job shop scheduling problems using Sheep Flocks Heredity Modal Algorithm," International Journal of Manufacturing Sciences and Technology, vol. 9, no. 2, pp. 47-54, March 2007.

[2] M. Eusuff, K. Lansey, and F. Pasha, "Shuffled frog-leaping algorithm: a memetic meta-heuristic for discrete optimization," Engineering Optima , vol. 38, pp. 129-154, 2006.

[3] H. Ohta and T. Nakatani, "A heuristic job-shop scheduling algorithm to minimize the total holding cost of completed and in-process products subject to no tardy jobs," Int. Jou. Of Prod. Eco., vol. 101, pp. 19-29, 2006.

[4] K. Nara, T. Takeyama, and H. Kim, "A New Evolutionary Algorithm based on Sheep Flocks Heredity Model and Its Application to thermal generator maintenance scheduling problem," IEEE Trans, vol. 6, pp. 503-508, 1999.

[5] S. Lawrence, Supplement to Resource Constrained Project Scheduling An Experimental Investigation of Heuristic Scheduling Techniques, Carnegie Mellon University, GSIA ,1984.

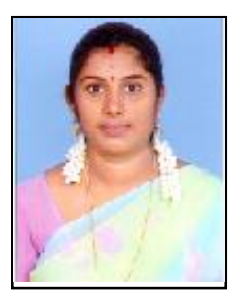

G. Ramya is a Reseach Scholar in Sathyabama University, Chennai, born on $19^{\text {th }}$ July 1982 . She has completed her UG B.E., Degree on Mechanical Engineering in Periyar University in the year May 2003. Then She has completed her PG M.E., Degree on CAD/CAM in Anna University in the year June 2007 and also she have been pursuing her Ph.D Research Work in the field Computer Integrated Manufacturing at Sathyabama University, Chennai, Tamilnadu, India. She have $71 / 2$ years teaching experience. She worked as LECTURER in Muthayammal polytechnic, Rasipuram from June 2003 to July 2005. Now, She is working as ASSISTANT PROFESSOR in Department of Mechanical Engineering, Rajalakshmi Engineering College from June 2007 to till date. She published many papers in International Conferences and Two International Journals.

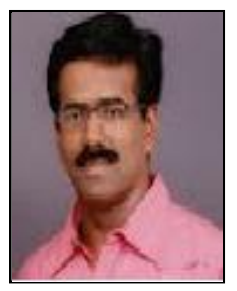

Dr. M. Chandrasekaran is working as a Professor and Director in Vels University. He has fifteen years of teaching experience in reputed colleges. $\mathrm{H}$ completed his Master's and Doctorate degree in Production Engineering at National Institute of Technology, Trichy, India. His research area includes Computer Integrated Manufacturing, Composites and Scheduling. He published many papers in International Journals and Conferences. He is an active member in ASME, ISTE, NIQR and Institution of Engineers Professional Societies. 